\title{
Dispersion management in highly nonlinear, carbon disulfide filled holey fibres
}

\author{
F. Poletti, A. Camerlingo, P. Petropoulos, and D. J. Richardson \\ Optoelectronics Research Centre, University of Southampton, Southampton, SO17 1BJ, United Kingdom \\ Email: frap@orc.soton.ac.uk
}

\begin{abstract}
We investigate dispersion control in holey fibres incorporating a highly nonlinear liquid. A liquid-core microstructured fibre with extremely high nonlinearity and flat dispersion at telecoms wavelengths is demonstrated.
\end{abstract}

\section{Introduction}

A large number of devices for all-optical processing at telecom wavelengths rely on nonlinear optical effects that take place along an optical fibre. To reduce the power or fibre length requirement, fibres with as high a nonlinearity $(\gamma)$ as is possible are required and for many applications, e.g. those based on four wave mixing, a low and flat group velocity dispersion (D) is desirable, if not essential. The current state-of-the art is represented by conventional highly nonlinear fibres, offering very low dispersion slope (DS) in the C+L band [1] and by silica holey fibres (HFs), which allow a flat dispersion over an even larger wavelength range [2]. In both cases however the maximum achievable $\gamma$ is limited to only 10 to 30 times that of a standard SMF, and therefore hundreds meters of fibre are generally required to achieve the desired nonlinear phase shift. Recent studies demonstrated that by using highly nonlinear liquids (HNLLs) in combination with nano-tapers or HFs extremely high values of $\gamma$ can be achieved [3, 4]; however in these works the dispersive properties were not optimised in any way.

In this study we explore how different glass-based, wavelength-size waveguides can be used in combination with a HNLL to generate the highest possible $\gamma$, while maintaining a low and flat dispersion in the $1.5-1.6 \mu \mathrm{m}$ range.

\section{Waveguide structures and optimum designs}

Among the many HNLLs that are available for nonlinear optic experiments, we here focus on carbon disulfide $\left(\mathrm{CS}_{2}\right)$ due to its high nonlinear refractive index $\left(\mathrm{n}_{2} \sim 3.2 \times 10^{-18}\right.$ for picosecond pulses [5]) and at the same time a good transmittivity in the near infrared [4]. Our analysis is restricted to waveguides based on the (modified) total internal reflection mechanism; hollow core bandgap fibres have not been considered since they would not allow the required low and flat dispersion in the region of interest, and ARROW-based fibres have been ignored since the mode inside the bandgap would be predominantly guided in the glass, thereby losing the benefit of the high $\mathrm{n}_{2}$ of the liquid.

The linear refractive index of $\mathrm{CS}_{2}$ at $1550 \mathrm{~nm}$ is $\sim 1.59$. We consider glasses with both a higher refractive index (bismuth oxide (Bi), $\mathrm{n} \sim 2.02, \mathrm{n}_{2} \sim 32 \times 10^{-20}$ and lead silicate (LS), $\mathrm{n} \sim 1.80, \mathrm{n}_{2} \sim 41 \times 10^{-20}$ ) and a lower refractive index (silica, $\mathrm{n} \sim 1.44, \mathrm{n}_{2} \sim 2.3 \times 10^{-20}$ ) than $\mathrm{CS}_{2}$. The three main cases we analyse are shown in Fig.1: (a) high refractive glass rod surrounded by $\mathrm{CS}_{2}$; (b) high refractive index $\mathrm{HF}$, infiltrated with $\mathrm{CS}_{2}$; (c) a silica $\mathrm{HF}$ with a hollow core, selectively filled with $\mathrm{CS}_{2}$. Simulations have been carried out with a full vector finite element method, while dispersion optimisation was obtained using a simplex method. The best dispersion curves are shown in Fig. 2.

(a)

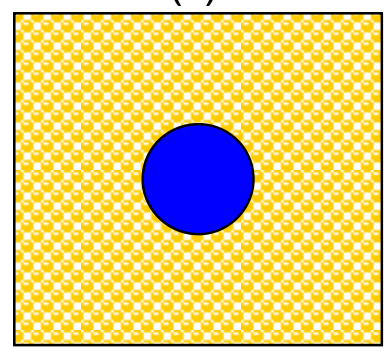

HNL Liquid (b)

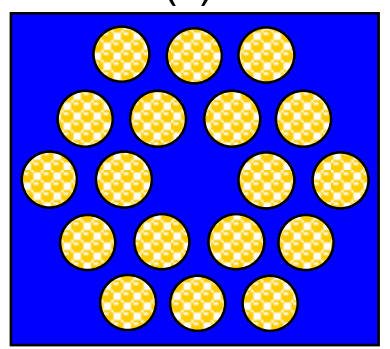

Soft glass (c)

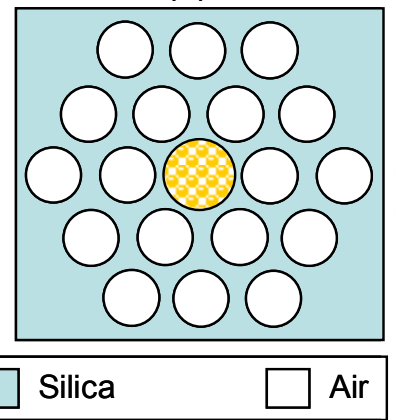

(d)

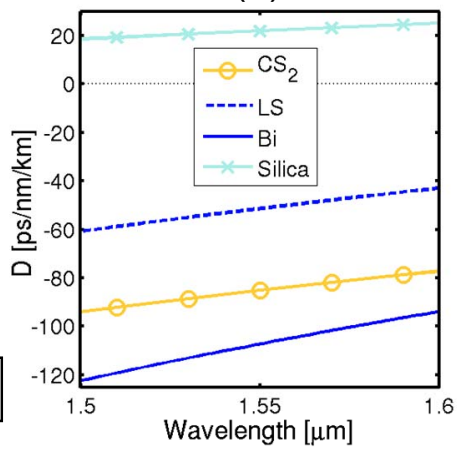

Fig.1. Structures studied: (a) soft glass rod immersed in liquid; (b) soft glass HF infiltrated with liquid; (c) silica HF with a central hollow core, liquid filled. (d) group velocity dispersion of all the materials employed in the study. 

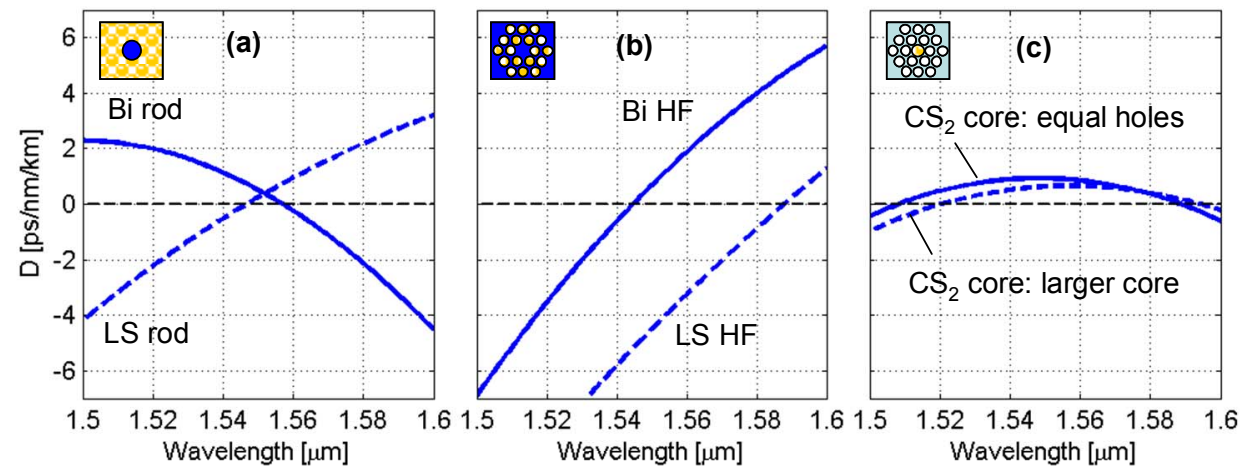

Fig.2. Best dispersion curves for the 3 cases (a), (b) and (c) shown in Fig.1

(a) Soft glass rod immersed in $\mathrm{CS}_{2}$. The single degree of freedom (core radius r) of this simple waveguide only allows a limited control over the achievable D. Using the parameters shown in Table 1, the best rod design provides a zero dispersion wavelength (ZDW) at $\sim 1550 \mathrm{~nm}$ with a relatively small DS of $\sim \pm 0.07 \mathrm{ps} / \mathrm{nm}^{2} / \mathrm{km}$ (Fig. 1(a)). The reduction in $\Delta \mathrm{n}$ from the glass-air case, and the need to control $\mathrm{D}$, reduce the amount of evanescent field in the HNLL to only $\sim 5 \%$ (LS) and $\sim 8 \%$ (Bi). Therefore $\gamma$ is only increased by $\sim 3 \%$ (LS) and $\sim 10 \%$ (Bi) with respect to the glass-air case. The fragility/long-term durability of such small rods, and the limited lengths that can be fabricated are other issues of concern, which can be mitigated by using a filled microstructure, as in case (b).

(b) Soft glass HF filled with $C S_{2}$. Despite the additional degree of design freedom we found that we were less able to control the net dispersion at $1550 \mathrm{~nm}$ for realistic core designs. The best $\mathrm{D}$ curves obtained are shown in Fig. 2(b). For Bi glass a ZDW at $1550 \mathrm{~nm}$ can still be obtained, although with nearly twice the absolute value of DS than in case (a). For the lower index LS glass, the waveguide dispersion is insufficient to completely compensate for the material dispersion. In both cases we have assumed a maximum realistically achievable hole size of $0.92 \Lambda$ due to fabrication constraints. As in case (a), most of the power resides in the glass, and the benefit of using the HNLL is limited to a slight increase in $\gamma(\sim 1-2 \%)$.

(c) Silica HF with a core selectively filled with $\mathrm{CS}_{2}$. First we studied the case with equal-sized holes. Fig.2(c) shows that with the optimum choice of the surrounding holey cladding, extremely flat $D$ within $\pm 1 \mathrm{ps} / \mathrm{nm} / \mathrm{km}$ can be achieved between 1.5 and $1.6 \mu \mathrm{m}$. Since $\sim 65 \%$ of the power is now concentrated in the HNLL, $\gamma$ as high as $\sim 6800$ $\mathrm{W}^{-1} \mathrm{~km}^{-1}$ can be achieved, in a fibre that is also effectively single mode. Since most selective filling techniques rely on holes with different sizes, we then also optimised a fibre with a larger central hole. As can be seen in Fig.2(c) and Table 1, a highly nonlinear, dispersion flattened fibre with similar performances can still be obtained.

Table 1 - Summary of the optical properties of the best fibres

\begin{tabular}{|c|c|c|c|c|c|}
\hline & \multicolumn{4}{|c|}{ Properties at $1550 \mathrm{~nm}$} & \multirow{2}{*}{ Dimensions } \\
\hline & $\underset{[\mathrm{ps} / \mathbf{n m} / \mathbf{k m}]}{D}$ & $\begin{array}{c}\text { Properties at } \\
\text { DS } \\
{\left[\mathbf{p s} / \mathbf{n m}^{2} / \mathbf{k m}\right]}\end{array}$ & $\begin{array}{c}\gamma 0 \mathrm{~nm} \\
\gamma \\
{\left[\mathbf{W}^{-1} \mathbf{k m}^{-1}\right]}\end{array}$ & $\begin{array}{l}\text { Power in } \\
\text { core }(\%)\end{array}$ & \\
\hline Bi rod & 0.5 & -0.069 & 1221 & 92 & $\mathrm{r}=0.65 \mu \mathrm{m}$ \\
\hline LS rod & 0.2 & 0.073 & 546 & 95 & $\mathrm{r}=1.13 \mu \mathrm{m}$ \\
\hline $\mathrm{Bi} \mathrm{HF}$ & 0.7 & 0.125 & 827 & 95 & $\Lambda=1.39 \mu \mathrm{m} ; \mathrm{d} / \Lambda=0.92$ \\
\hline LS HF & -4.5 & 0.129 & 371 & 97 & $\Lambda=2.40 \mu \mathrm{m} ; \mathrm{d} / \Lambda=0.92$ \\
\hline $\mathrm{SiO}_{2} \mathrm{HF}$ (equal holes) & 0.9 & -0.002 & 6776 & 65 & $\Lambda=1.36 \mu \mathrm{m} ; \mathrm{d} / \Lambda=0.75$ \\
\hline $\mathrm{SiO}_{2} \mathrm{HF}$ (larger central hole) & 0.6 & 0.007 & 6548 & 75 & $\Lambda=1.36 \mu \mathrm{m} ; \mathrm{dc} / \Lambda=0.9 ; \mathrm{d} / \Lambda=0.65$ \\
\hline
\end{tabular}

\section{Conclusion}

We studied various arrangements for obtaining ultra-high $\gamma$ in a dispersion flattened fibre using a HNLL. Configurations with a soft glass core allow some D control, but do not fully exploit the high liquid nonlinearity. The best result was obtained for a liquid filled core HF, where external air holes contribute to tailor the fibre's dispersion. The ultimate loss of such a fibre and suitable selective filling strategies are currently under investigation.

\section{References}

[1] M. Takahashi et al., "Low-loss and low-dispersion-slope highly nonlinear fibers", J. Lightwave Technol., 23, 3615-3624 (2005)

[2] W. H. Reeves et al., "Demonstration of ultra-flattened dispersion in photonic crystal fibers", Opt. Expr., 10, 609-613 (2002)

[3] K. Rowland et al., "Nonlinearity enhancement of filled microstructured fibers operating in the nanowire regime”, OFC 2006 , paper OTuH3.

[4] R. Zhang et al., "Theoretical design of a liquid-core photonic crystal fiber for supercontinuum generation", Opt. Expr., 14, 6800-6812 (2006)

[5] R. A. Ganeev et al., "Nonlinear optical characteristics of carbon disulfide”, Optics and Spectroscopy, 100, 108-118 (2006). 